\title{
On the Existence of Equilibria in
}

\section{Discontinuous Games: Three Counterexamples*}

\author{
Guilherme Carmona
}

Universidade Nova de Lisboa

Faculdade de Economia

Campus de Campolide, 1099-032 Lisboa, Portugal

email: gcarmona@fe.unl.pt

telephone: (351) 213801671

fax: (351) 213886073

August 13, 2004

\footnotetext{
${ }^{*}$ I wish to thank the editor, an associate editor and an anonymous referee for very helpful comments. I thank also John Huffstot for editorial assistance. Any remaining error is, of course, mine.
} 


\begin{abstract}
We study whether we can weaken the conditions given in Reny [4] and still obtain existence of pure strategy Nash equilibria in quasiconcave normal form games, or, at least, existence of pure strategy $\varepsilon$-equilibria for all $\varepsilon>0$. We show by examples that there are:

1. quasiconcave, payoff secure games without pure strategy $\varepsilon$-equilibria for small enough $\varepsilon>0$ (and hence, without pure strategy Nash equilibria),

2. quasiconcave, reciprocally upper semicontinuous games without pure strategy $\varepsilon$-equilibria for small enough $\varepsilon>0$, and

3. payoff secure games whose mixed extension is not payoff secure. The last example, due to Sion and Wolfe [6], also shows that nonquasiconcave games that are payoff secure and reciprocally upper semicontinuous may fail to have mixed strategy equilibria.
\end{abstract}

Keywords: Discontinuous games, payoff security, reciprocal upper semicontinuity, Nash equilibrium. 


\section{Introduction}

Recently, there has been an attempt to extend Nash's existence result [3] from finite normal form games to infinite-action games with discontinuous payoff functions (see Baye et al. [1], Dasgupta and Maskin [2], Reny [4] and Simon [5]). The most general result is due to Reny [4], who showed that a quasiconcave normal form game has a pure strategy Nash equilibrium if it is better-reply secure.

Better-reply security combines and generalizes two intuitive conditions, payoff security ${ }^{1}$ and reciprocal upper semicontinuity. ${ }^{2}$ Our goal is to study whether any such condition alone is enough to guarantee, in quasiconcave games, the existence of pure strategy Nash equilibria or, at least, the existence of pure strategy $\varepsilon$-equilibria, for all $\varepsilon>0$.

We provide two examples that show that we cannot drop either of the two conditions and still obtain a pure strategy Nash equilibrium. Both games in the examples are simple games, games in which each player's payoff function

\footnotetext{
1 "Payoff security requires that for all strategies $x$, each player has a strategy that virtually guarantees the payoff he receives at $x$, even if the others deviate slightly from $x$ " (Reny [4]).

2 "Reciprocal upper semicontinuity requires that some player's payoff jumps up whenever some other player's payoff jumps down" (Reny [4]).
} 
is simple (i.e., has a finite range). Since a simple game has a Nash equilibrium if and only if it has $\varepsilon$-equilibria for all $\varepsilon>0$ (see Proposition 2), our examples also show that neither payoff security nor reciprocal upper semicontinuity alone is enough to guarantee the existence of pure strategy $\varepsilon$-equilibrium, for all $\varepsilon>0$, in quasiconcave games.

When applied to the mixed extension of a normal form game, Reny's Theorem asserts that a mixed strategy equilibrium exists for a given normal form game provided that its mixed extension is payoff secure and reciprocally upper semicontinuous. It is useful to know when we can conclude that the mixed extension of a given normal form game satisfies those conditions by studying the properties of the original game, since the analysis of the latter is typically easier than that of its mixed extension. In this line of research, Reny showed that a sufficient condition for the mixed extension of a given normal form game to be reciprocally upper semicontinuous is that the sum of the payoff functions of the original game is upper semicontinuous.

Given the above result, we ask whether the payoff security of the mixed extension of a given normal form game follows from the payoff security of the original game. We use the example in Sion and Wolfe [6] to show that this conjecture is false. This implies that non-quasiconcave, payoff secure, 
reciprocally upper semicontinuous games may fail to have mixed strategy equilibria.

\section{Normal form games}

A normal form game $G$ consists of a finite set of players $N=\{1, \ldots, n\}$, and, for all players $i \in N$, a pure strategy set $X_{i}$, represented by a nonempty compact subset of a topological vector space, and a bounded payoff function $U_{i}: X \rightarrow \mathbb{R}$, where $X=\times_{i \in N} X_{i}$. A normal form game is simple if for all $i \in N, U_{i}$ is a simple function (i.e., a function with the property that its range is a finite set).

Throughout, the product of any number of sets is endowed with the product topology. Given a player $i \in N$, the symbol $-i$ denotes "all players but i." Also, $X_{-i}=\times_{j \neq i} X_{j}$.

The vector of the players' payoff functions is denoted by $U: X \rightarrow \mathbb{R}^{N}$ and is defined by $U(x)=\left(U_{1}(x), \ldots, U_{n}(x)\right)$ for all $x \in X$. The graph of $U$ is the subset of $X \times \mathbb{R}^{n}$ given by $\left\{(x, u) \in X \times \mathbb{R}^{n}: u=U(x)\right\}$. It is denoted by $\operatorname{graph}(U)$.

Given a player $i \in N$, player $i$ can secure a payoff of $\alpha \in \mathbb{R}$ at $x \in X$ if 
there exists $\tilde{x}_{i} \in X_{i}$ and a neighborhood $V_{x_{-i}}$ of $x_{-i}$ such that $U_{i}\left(\tilde{x}_{i}, x_{-i}^{\prime}\right) \geq \alpha$ for all $x_{-i}^{\prime} \in V_{x_{-i}}$ (see Reny [4]).

A payoff function $U_{i}$ for player $i \in N$ is payoff secure if for all $x \in X$ and all $\varepsilon>0$, player $i$ can secure a payoff of $U_{i}(x)-\varepsilon$ at $x$ (see Reny [4]). A normal-form game $G=\left\langle N,\left(X_{i}, U_{i}\right)_{i \in N}\right\rangle$ is payoff secure if for all $i \in N, U_{i}$ is payoff secure.

A game $G=\left\langle N,\left(X_{i}, U_{i}\right)_{i \in N}\right\rangle$ is reciprocally upper semicontinuous if for all $(x, u) \in \overline{\operatorname{graph}(U)}$ such that $U_{i}(x) \leq u_{i}$ for all $i \in N$, then $U_{i}(x)=u_{i}$ for all $i \in N$ (see Simon [5] and Reny [4]). ${ }^{3}$

A game $G=\left\langle N,\left(X_{i}, U_{i}\right)_{i \in N}\right\rangle$ is quasiconcave if for all $i \in N, X_{i}$ is convex and $U_{i}\left(\cdot, x_{-i}\right)$ is quasiconcave on $X_{i}$, for all $x_{-i} \in X_{-i}$.

If $X_{i}$ is a compact Hausdorff space, let $M_{i}$ be the set of all regular probability measures in $X_{i}$ and $M=\times_{i \in N} M_{i}$. If $U_{i}$ is Borel measurable, we define $v_{i}: M \rightarrow \mathbb{R}$ by

$$
v_{i}(\mu)=\int_{X} U_{i} \mathrm{~d} \mu .
$$

Finally, we define the mixed extension of $G$ to be $\bar{G}=\left\langle N,\left(M_{i}, v_{i}\right)_{i \in N}\right\rangle$.

Given a normal form game $G=\left\langle N,\left(X_{i}, U_{i}\right)_{i \in N}\right\rangle$, a pure strategy Nash

\footnotetext{
${ }^{3}$ For all subsets $A$ of a topological space $Y, \bar{A}$ denotes the closure of $A$.
} 
equilibrium of $G$ is $x^{*} \in X$ such that, for all $i \in N$ and $x_{i} \in X_{i}$,

$$
U_{i}\left(x^{*}\right) \geq U_{i}\left(x_{i}, x_{-i}^{*}\right)
$$

Given $\varepsilon>0$, a pure strategy $\varepsilon$-equilibrium of $G$ is $x^{*} \in X$ such that, for all $i \in N$ and $x_{i} \in X_{i}$

$$
U_{i}\left(x^{*}\right) \geq U_{i}\left(x_{i}, x_{-i}^{*}\right)-\varepsilon .
$$

A mixed strategy Nash equilibrium of $G$ is a pure strategy Nash equilibrium of $\bar{G}$, and a mixed strategy $\varepsilon$-equilibrium of $G$ is a pure strategy $\varepsilon$-equilibrium of $\bar{G}$.

\section{On the Existence of Nash Equilibria}

The following is an example of a simple, quasiconcave and payoff secure game that has no pure strategy Nash equilibrium. Since a simple game has a pure strategy Nash equilibrium if and only if it has an $\varepsilon$-equilibrium for all $\varepsilon>0$

(Proposition 2), we conclude that payoff security is not enough to guarantee the existence of pure strategy $\varepsilon$-equilibrium, for all $\varepsilon>0$, in quasiconcave games.

Example 1 Let $G_{1}$ be described by $N=\{1,2\}, X_{1}=X_{2}=[0,1], U_{1}: X \rightarrow$ 

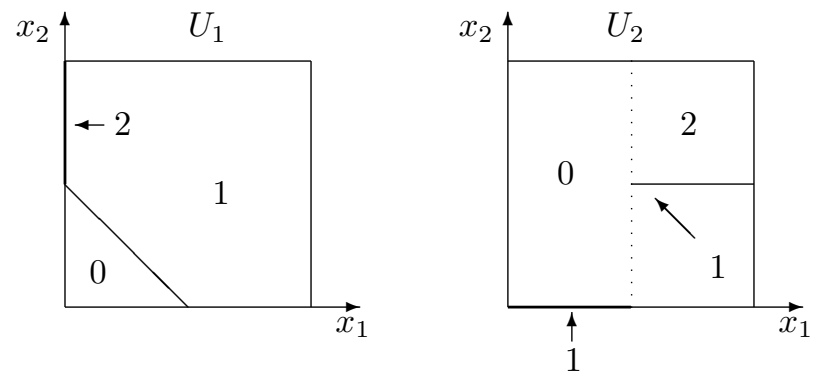

Figure 1: The utility functions from Example 1. The numbers denote the utility levels in the indicated areas

$\mathbb{R}$ be defined by

$$
U_{1}\left(x_{1}, x_{2}\right)= \begin{cases}0 & \text { if } x_{2} \leq \frac{1}{2}-x_{1} \\ 2 & \text { if } x_{1}=0 \text { and } x_{2}>\frac{1}{2} \\ 1 & \text { otherwise, }\end{cases}
$$

and $U_{2}: X \rightarrow \mathbb{R}$ be defined by

$$
U_{2}\left(x_{1}, x_{2}\right)= \begin{cases}0 & \text { if } x_{1} \leq \frac{1}{2} \text { and } x_{2}>0 \\ 1 & \text { if } x_{1} \leq \frac{1}{2} \text { and } x_{2}=0 \\ 1 & \text { if } x_{1}>\frac{1}{2} \text { and } x_{2} \leq \frac{1}{2} \\ 2 & \text { if } x_{1}>\frac{1}{2} \text { and } x_{2}>\frac{1}{2}\end{cases}
$$

Proposition 1 The game $G_{1}$ is quasiconcave and payoff secure but has no pure strategy $\varepsilon$-equilibrium, if $\varepsilon>0$ is small enough. In particular, $G_{1}$ has 
no pure strategy Nash equilibrium.

Proof. One easily checks that $G_{1}$ is quasiconcave. We show next that $G_{1}$ is payoff secure. To show that $U_{1}$ is payoff secure, it is enough to show that $U_{1}$ is payoff secure at $\left(x_{1}, x_{2}\right)$ satisfying $x_{1}=0$ and $x_{2}>1 / 2$. This is so because at points $x$ at which $U_{1}(x)=0$ there is nothing to show, and in the remaining case, i.e., when $U_{1}(x)=1, U_{1}$ is continuous. If $x_{1}=0$ and $x_{2}>1 / 2$ then $U_{1}\left(x_{1}, x_{2}\right)=2$. Let $\tilde{x}_{1}=0$ and $V_{x_{2}}$ be the open ball centered at $x_{2}$ with radius $\delta=x_{2}-1 / 2$. Then, $U_{1}\left(\tilde{x}_{1}, x_{2}^{\prime}\right)=2$ for all $x_{2}^{\prime} \in V_{x_{2}}$. Similarly for $U_{2}$ it is enough to consider the following two cases: the first is when $x_{2}=0$ and $0 \leq x_{1} \leq 1 / 2$, in which case we let $\tilde{x}_{2}=0$; and the second is when $x_{2}=1 / 2$ and $x_{1}>1 / 2$, in which case we let $\tilde{x}_{2}=1 / 2$ and $V_{x_{1}}$ be the open ball centered at $x_{1}$ with radius $\delta=x_{1}-1 / 2$. Hence, $G_{1}$ is payoff secure.

Letting $\beta_{i}$ denote player $i$ 's best reply correspondence, we obtain

$$
\beta_{1}\left(x_{2}\right)= \begin{cases}\left(\frac{1}{2}-x_{2}, 1\right] & \text { if } x_{2} \leq \frac{1}{2} \\ \{0\} & \text { if } x_{2}>\frac{1}{2} .\end{cases}
$$

and

$$
\beta_{2}\left(x_{1}\right)= \begin{cases}\{0\} & \text { if } x_{1} \leq \frac{1}{2} \\ \left(\frac{1}{2}, 1\right] & \text { if } x_{1}>\frac{1}{2}\end{cases}
$$


Since the graph of $\beta_{1}$ does not intersect the graph of $\beta_{2}$, it follows that there is no pure strategy Nash equilibrium. Also, we conclude from Proposition 2 that $G$ has no pure strategy $\varepsilon$-equilibrium, if $\varepsilon>0$ is small enough.

As claimed above, for simple games the existence of Nash equilibria is equivalent to the existence of $\varepsilon$-equilibria for all $\varepsilon>0$.

Proposition 2 Let $G=\left\langle N,\left(X_{i}, U_{i}\right)_{i \in N}\right\rangle$ be a simple game. Then, $G$ has a pure strategy Nash equilibrium if and only if $G$ has a pure strategy $\varepsilon-$ equilibrium, for all $\varepsilon>0$.

Proof. Since necessity is obvious, we prove only sufficiency.

For all $i \in N$, let $U_{i}(X)=\left\{d_{1}^{i}, \ldots, d_{L_{i}}^{i}\right\}$, with $d_{1}^{i}<\ldots<d_{L_{i}}^{i}$ and let $\varepsilon>0$ be such that $\varepsilon<\min _{i \in N} \min _{l \in\left\{1, \ldots, L_{i}-1\right\}}\left(d_{l+1}^{i}-d_{l}^{i}\right)$. Let $x^{*} \in X$ be an $\varepsilon-$ equilibrium. Given the way $\varepsilon$ was constructed, it follows that $U_{i}\left(x^{*}\right)+\varepsilon \geq$ $U_{i}\left(x_{i}, x_{-i}^{*}\right)$, for all $x_{i} \in X_{i}$, implies $U_{i}\left(x^{*}\right) \geq U_{i}\left(x_{i}, x_{-i}^{*}\right)$, for all $x_{i} \in X_{i}$. Thus, $x^{*}$ is a Nash equilibrium of $G$.

The following is an example of a simple, quasiconcave and reciprocally upper semicontinuous game that has no pure strategy Nash equilibrium. Hence, reciprocally upper semicontinuity is not enough to guarantee the existence of pure strategy $\varepsilon$-equilibrium in quasiconcave games. 

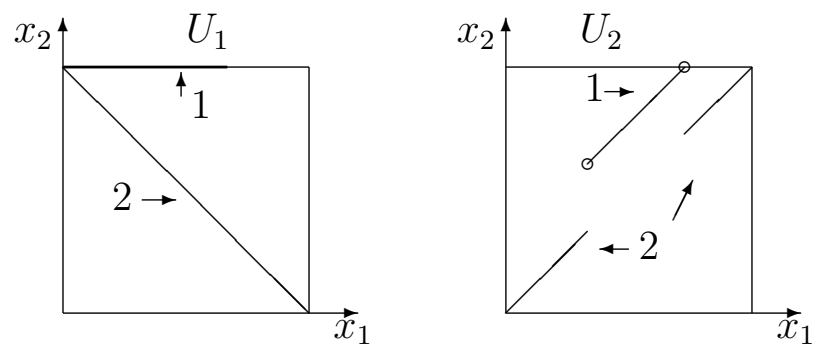

Figure 2: The utility functions from Example 2.

Example 2 Let $G_{2}$ be described by $N=\{1,2\}, X_{1}=X_{2}=[0,1], U_{1}: X \rightarrow$ $\mathbb{R}$ be defined by

$$
U_{1}\left(x_{1}, x_{2}\right)= \begin{cases}2 & \text { if } x_{2}=1-x_{1} \\ 1 & \text { if } 0<x_{1} \leq \frac{2}{3} \text { and } x_{2}=1 \\ 0 & \text { otherwise, }\end{cases}
$$

and $U_{2}: X \rightarrow \mathbb{R}$ be defined by

$$
U_{2}\left(x_{1}, x_{2}\right)= \begin{cases}2 & \text { if } x_{1} \leq \frac{1}{3} \text { and } x_{2}=x_{1} \\ 2 & \text { if } x_{1} \geq \frac{2}{3} \text { and } x_{2}=x_{1} \\ 1 & \text { if } \frac{1}{3}<x_{1}<\frac{2}{3} \text { and } x_{2}=\frac{1}{3}+x_{1} \\ 0 & \text { otherwise. }\end{cases}
$$

Proposition 3 The game $G_{2}$ is quasiconcave and reciprocally upper semicontinuous but has no pure strategy $\varepsilon$-equilibrium, if $\varepsilon>0$ is small enough. 
In particular, $G_{2}$ has no pure strategy Nash equilibrium.

Proof. One easily shows that $G_{2}$ is quasiconcave. We show below that $G_{2}$ is reciprocally upper semicontinuous. For convenience, let $\left\{U_{1}+U_{2} \geq \gamma\right\}$ denote

$$
\left\{\left(x_{1}, x_{2}\right) \in[0,1] \times[0,1]: U_{1}\left(x_{1}, x_{2}\right)+U_{2}\left(x_{1}, x_{2}\right) \geq \gamma\right\},
$$

for all $\gamma \in \mathbb{R}$. Let $\alpha \in \mathbb{R}$. We have that

$$
\left\{U_{1}+U_{2} \geq \alpha\right\}= \begin{cases}\emptyset & \text { if } \alpha>2, \\ \left\{U_{1}+U_{2} \geq 2\right\} & \text { if } 1<\alpha \leq 2 \\ \left\{U_{1}+U_{2} \geq 1\right\} & \text { if } 0<\alpha \leq 1 \\ X & \text { otherwise. }\end{cases}
$$

Since

$$
\begin{aligned}
\left\{U_{1}+U_{2} \geq 2\right\}= & \left\{\left(x_{1}, x_{2}\right): x_{1}+x_{2}=1\right\} \cup \\
& \left\{\left(x_{1}, x_{2}\right): x_{1}=x_{2} \text { and } x_{1} \leq 1 / 3\right\} \cup \\
& \left\{\left(x_{1}, x_{2}\right): x_{1}=x_{2} \text { and } x_{1} \geq 2 / 3\right\}
\end{aligned}
$$

and

$$
\begin{aligned}
\left\{U_{1}+U_{2} \geq 1\right\}= & \left\{U_{1}+U_{2} \geq 2\right\} \cup\left\{\left(x_{1}, x_{2}\right): x_{1} \leq 2 / 3 \text { and } x_{2}=1\right\} \cup \\
& \left\{\left(x_{1}, x_{2}\right): x_{2}=1 / 3+x_{1} \text { and } 1 / 3 \leq x_{1} \leq 2 / 3\right\}
\end{aligned}
$$


it follows that both $\left\{U_{1}+U_{2} \geq 2\right\}$ and $\left\{U_{1}+U_{2} \geq 1\right\}$ are closed. Hence, $U_{1}+U_{2}$ is upper semicontinuous and $G_{2}$ is reciprocally upper semicontinuous. Letting $\beta_{i}$ denote player $i$ 's best reply correspondence, we obtain

$$
\beta_{1}\left(x_{2}\right)=\left\{1-x_{2}\right\}
$$

for all $x_{2} \in X_{2}$ and

$$
\beta_{2}\left(x_{1}\right)= \begin{cases}\left\{\frac{1}{3}+x_{1}\right\} & \text { if } \frac{1}{3}<x_{1}<\frac{2}{3} \\ \left\{x_{1}\right\} & \text { otherwise. }\end{cases}
$$

Since the graph of $\beta_{1}$ does not intersect the graph of $\beta_{2}$, it follows that there is no pure strategy Nash equilibrium. Hence, $G$ has no pure strategy $\varepsilon$-equilibrium, if $\varepsilon>0$ is small enough.

Our third and last example answers the question of whether or not the payoff security of the mixed extension $\bar{G}$ of a normal form game $G$ follows from the payoff security of $G$. It shows that a game can be payoff secure in pure strategies without being payoff secure in mixed strategies.

Example 3 (Sion and Wolfe [6]) Let $G_{3}$ be described by $N=\{1,2\}, X_{1}=$ $X_{2}=[0,1], U_{1}: X \rightarrow \mathbb{R}$ be defined by

$$
U_{1}\left(x_{1}, x_{2}\right)= \begin{cases}-1 & \text { if } x_{1}<x_{2}<x_{1}+\frac{1}{2} \\ 0 & \text { if } x_{1}=x_{2} \text { or } x_{2}=x_{1}+\frac{1}{2} \\ 1 & \text { otherwise }\end{cases}
$$




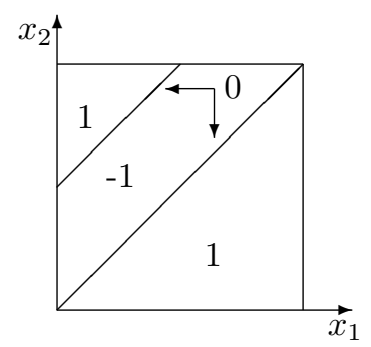

Figure 3: The function $U_{1}$ from Example 3.

and $U_{2}: X \rightarrow \mathbb{R}$ be defined by $U_{2}\left(x_{1}, x_{2}\right)=-U_{1}\left(x_{1}, x_{2}\right)$, for all $\left(x_{1}, x_{2}\right) \in X$.

Proposition 4 The game $G_{3}$ is payoff secure but its mixed extension $\bar{G}_{3}$ is not.

Proof. One easily checks that $G_{3}$ is payoff secure. For player 1 , it is enough to consider $x \in X$ such that $U_{1}(x)=0$ since when $U_{1}(x)=-1$ there is nothing to show, and $U_{1}$ is continuous in the remaining case (i.e., for $x$ such that $U_{1}(x)=1$ ). If $x_{1}=x_{2}$, we let $\tilde{x}_{1}=1$; if $x_{2}=x_{1}+1 / 2$, we let $\tilde{x}_{1}=0$ except when $x_{1}=1 / 2$, in which case we let $\tilde{x}_{1}=1$. In all these cases we can find a neighborhood $V_{x_{2}}$ of $x_{2}$ such that $U_{1}\left(\tilde{x}_{1}, x_{2}^{\prime}\right)=1$ for all $x_{2}^{\prime} \in V_{x_{2}}$. 
For player 2, it is again enough to consider $x \in X$ such that $U_{1}(x)=0$. We let $\tilde{x}_{2}=\min \left\{x_{1}+1 / 4,1\right\}$. In all these cases we can find a neighborhood $V_{x_{1}}$ of $x_{1}$ such that $U_{2}\left(x_{1}^{\prime}, \tilde{x}_{2}\right)=1$ for all $x_{1}^{\prime} \in V_{x_{1}}$.

Also, since $G_{3}$ is a zero-sum game, so is $\bar{G}_{3}$; hence, $\bar{G}_{3}$ is reciprocally upper semicontinuous. However, as Sion and Wolfe [6] have shown, $G_{3}$ has no Nash equilibrium (pure or mixed), and so it follows from Reny's Theorem that $\bar{G}_{3}$ is not payoff secure.

Sion and Wolfe [6] have shown that $G_{3}$ has no Nash equilibrium (pure or mixed); hence, $G_{3}$ is also an example of a (non-quasiconcave) reciprocally upper semicontinuous and payoff secure game without Nash equilibria.

\section{References}

[1] Baye, M., G. Tian, and J. Zhou (1993), "Characterization of the Existence of Equilibria in Games with Discontinuous and Non-quasiconcave Payoff," Review of Economics Studies, 60, 935-948.

[2] Dasgupta, P. and E. Maskin, (1986), "The Existence of Equilibrium in Discontinuous Economic Games, I: Theory," Review of Economics Studies, 53, 1-26. 
[3] Nash, J. (1950), "Equilibrium Points in n-Person Games," Proceedings of the National Academy of Science, 36, 170-174.

[4] Reny, P. (1999) "On the Existence of Pure and Mixed Strategy Equilibria in Discontinuous Games," Econometrica, 67, 1029-1056.

[5] Simon, L., (1987), "Games with Discontinuous Payoffs," Review of Economic Studies, 54, 569-597.

[6] Sion, M. and P. Wolfe, (1957), "On a Game without a Value," in Contributions to the Theory of Games, volume III, edited by M. Dresher, A. W. Tucker, and P. Wolfe, Princeton: Princeton University Press. 